\title{
Effect of Ti and W Additions on the Microstructural Behavior of a Nanocrystalline CoCrFeMoNi High Entropy Alloy
}

\author{
C.D. Gómez-Esparza ${ }^{1}$, H. Camacho-Montes ${ }^{1}$, I. Estrada-Guel², R. Martínez-Sánchez ${ }^{2}$ and C.A. \\ Rodríguez-Gónzalez ${ }^{1}$ \\ 1. Instituto de Ingeniería y Tecnología, Universidad Autónoma de Ciudad Juárez (UACJ), Av. Del \\ Charro 450 Norte, 32310, Cd. Juárez, Chihuahua, México. \\ 2. Centro de Investigación en Materiales Avanzados (CIMAV), Laboratorio Nacional de \\ Nanotecnología, Miguel de Cervantes No. 120, 31136 Chihuahua, Chihuahua, México.
}

Over the past decade, a new alloy design, the high entropy alloys (HEAs), has attracted significant attention due to their unusual properties. Due to the fact that HEAs are composed of at least five principal elements, the compositional design is one of the most critical factors in the selection and development of these materials. The selection of chemical compositions of HEAs has been focused in the aim to obtain solid solution with simple structures, in most of the cases, single FCC or BCC solid solution phases [1].

In the other hand, the processing route is one of the main parameters to the resulting properties of HEAs. Mechanical alloying is a solid state powder processing technique to synthesized equilibrium and nonequilibrium alloys in nanometric scale with superior properties. The use of mechanical alloying in the production of HEAs sometimes comprises subsequent surface coatings techniques for different applications, where high hardness is needed.

In this investigation, a series of three CoCrFeNiMo, CoCrFeNiMoW and CoCrFeNiMoTi alloys is used to study the effect of composition on the microstructural behavior and hardness. The selection of this series of high entropy alloys is based on that equimolar CoCrFeNi system has been reported as a single FCC solid solution. In the other hand, the addition of refractory elements as Mo, Ti and W to tool steels improves temper softening resistance, surface hardness and wear resistance. HEAs are known to have good thermal stability, high hardness and strength and, excellent wear resistance [2]. The use of these kind of refractory elements to high entropy alloys suggests the improvement of hardness.

The ball milling was performed under argon atmosphere using SPEX 8000M high-energy mill during 10 h. Methanol was used to prevent adhesion of powder. The ball-to-powder weight ratio was 5:1. To avoid overheating, cycles of $1 \mathrm{~h}$ milling and $0.5 \mathrm{~h}$ rest were performed. The microstructural features of powder samples were characterized by a Hitachi SU 3500 scanning electron microscope, while structural characterization was carried out in a Panalytical X-Pert system. The determination of hardness was carried out by microhardness (HV) testing of polished cross-section powders specimens.

After milling, the CoCrFeNiMo alloy exhibit a particle size of 2-20 $\mu \mathrm{m}$; the addition of $\mathrm{W}$ reduces the particle size distribution $\left(\mathrm{d}_{0.5}=5 \mu \mathrm{m}\right)$, while Ti addition promotes an increase of particle size $(\leq 30 \mu \mathrm{m})$. According to XRD patterns, the three powder alloys are the mixture of body-centered cubic (BCC) and face-centered cubic (FCC) solid solution phases.

The Vickers microhardness values of 721,743 and $654 \mathrm{HV}$ were obtained from the CoCrFeNiMo, $\mathrm{CoCrFeNiMoW}$ and CoCrFeNiMoTi alloys, respectively. The HEA containing $\mathrm{W}$ is the hardest alloy, 
but only 3\% above the CoCrFeNiMo alloy [4].

References:

[1] M.C. Troparevsky, J.R. Morris, P.R.C. Kent, A.R. Lupini, G.M. Stocks, Phys. Rev. X 5 (2015) 011041.

[2] K. Kanayo-Alanemea, M. Oluwatosin-Bodunrina, S. Ranti-Oke, J. Mater. Res. Technol. 5 (2016), p. 384.

[3] The authors are grateful to the Laboratorio Nacional de Nanotecnología of the Centro de Investigación en Materiales Avanzados (CIMAV) for the technical assistance.
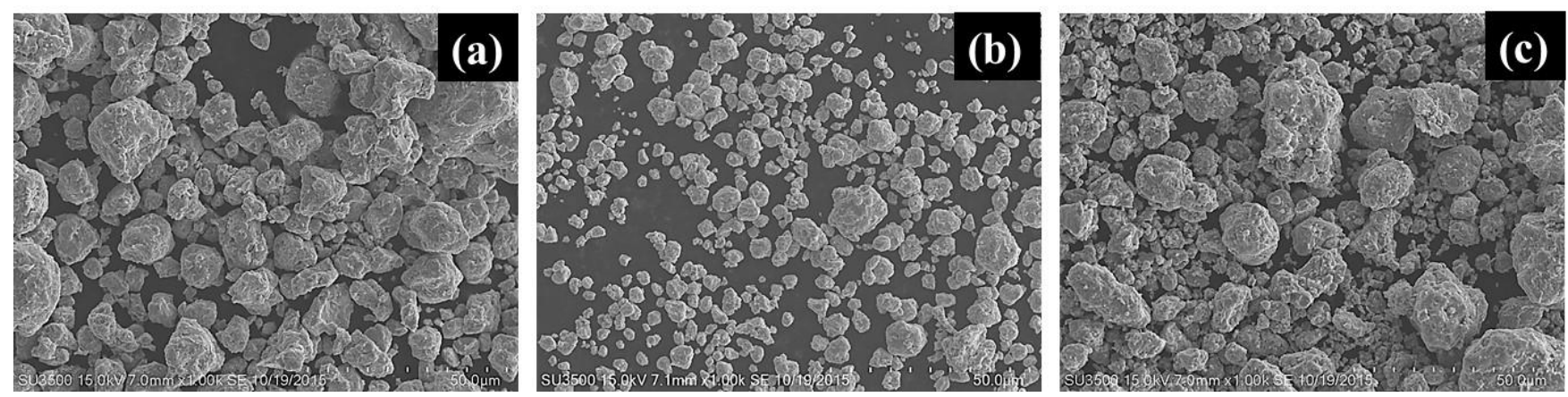

Figure 1. SEM-secondary electron images of as-milled: (a) CoCrFeNiMo, (b) CoCrFeNiMoW and (c) CoCrFeNiMoTi alloys.

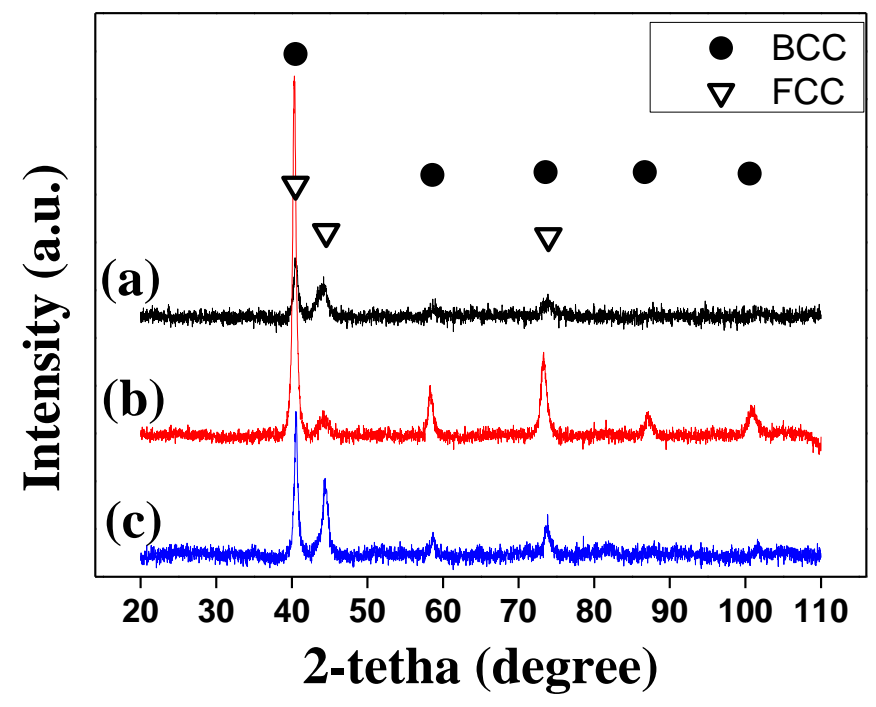

Figure 2. XRD patterns of: (a) CoCrFeNiMo, (b) CoCrFeNiMoW and (c) CoCrFeNiMoTi alloys after $10 \mathrm{~h}$ of milling. 